\title{
September 21: World Day of Alzheimer's Disease
}

\author{
Gabriel Miranda Nava* \\ Neurologist and Clinical Neurophysiologist, Head of service assigned to the Hospital Center of the Presidential General Staff, Mexico
}

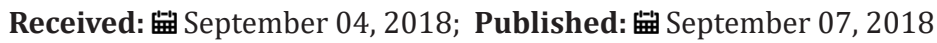

*Corresponding author: Gabriel Miranda Nava, Neurologist and Clinical Neurophysiologist, Head of service assigned to the Hospital Center of the Presidential General Staff, Mexico

\section{Opinion}

Before talking about Alzheimer's disease, we must understand the concept of dementia, which is conceptualized as the progressive loss of cognitive functions due to brain damage or disorders. Characteristically, this cognitive alteration causes inability to perform the activities of daily life mainly seen in memory, calculation, way of relating and living together, as well as in decision making. Many times, dementia is caused by other types of ailments, such as brain tumors, hydrocephalus, multiple cerebral infarcts, metabolic diseases such as hypothyroidism, chronic infections; or it can also be simulated by anxiety and chronic depression. Being the most frequent cause of dementia (although we already stressed that it is not the only one), Alzheimer's disease was discovered and reported in 1907 by the German Alois Alzheimer, and it is when until a few years ago it was of unusual characteristics due to the poor expectation of life, but by increasing this, a series of chronic degenerative diseases are derived at the same time, with an increase in their frequency of appearance, among them the condition that welcomes us today.

This degenerative dementia is characterized by a gradual onset, but irreversible at the same time, consisting of depression, anxiety, loss of memory, failure of judgment and calculation, view of the handling of money, unexpected exits of the patient from his home, repetition of ideas, as well as altered visions of reality and persecutory thoughts; this series of symptoms are increasingly frequent and intense, leading the patient to prostration, and being prey to respiratory or urinary tract infections, but not before altering the mental state of relatives and caregivers surrounding the patient. There are 40 million Alzheimer's patients in the world, and it will continue to rise, due to the investment of the age pyramid, and the expected increase in the duration and quality of life in people, through the new medical interventions They are discovering day by day. The drugs are often symptomatic and fail to effectively stop the clinical picture, often the basis of treatment is the patient's previous struggle, is the "store for the winter"; we refer to that we would face the eventual possibility of this disease through what we do in previous stages, and more specifically in a healthy lifestyle. A healthy lifestyle is always to have proper sleep habits, avoid smoking and drinking, have the habit of reading and always fortify our thoughts with education and culture, not live situations too stress, care and anticipate our economy with an adequate pension system and try to belong to a health system that has adequate coverage for problems of adults of the third age.

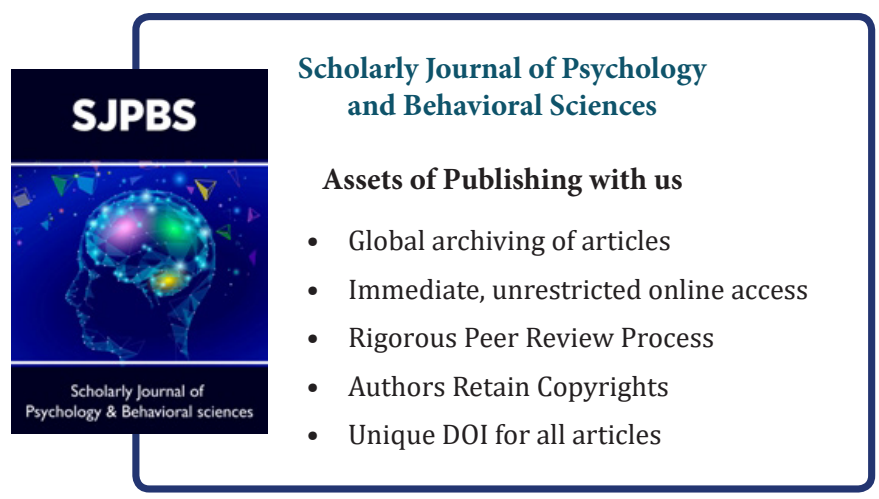

